\title{
Unusual Odd-Electron Fragments from Even-Electron Protonated Prodiginine Precursors Using Positive-Ion Electrospray Tandem Mass Spectrometry
}

\author{
Kan Chen, ${ }^{a}$ Nalaka S. Rannulu, ${ }^{\text {a }}$ Yang Cai, ${ }^{\text {a,b }}$ Pat Lane, ${ }^{a}$ Andrea L. Liebl, \\ Bernard B. Rees, ${ }^{\mathrm{c}}$ Christophe Corre, ${ }^{\mathrm{d}}$ Gregory L. Challis, ${ }^{\mathrm{d}}$ and \\ Richard B. Cole ${ }^{a}$ \\ a Department of Chemistry, University of New Orleans, New Orleans, Louisiana, USA \\ b The Research Institute for Children, Children's Hospital of New Orleans, New Orleans, Louisiana, USA \\ ${ }^{c}$ Department of Biological Sciences, University of New Orleans, New Orleans, Louisiana, USA \\ ${ }^{\mathrm{d}}$ Department of Chemistry, University of Warwick, Coventry, United Kingdom
}

Reports of anticancer and immunosuppressive properties have spurred recent interest in the bacterially produced prodiginines. We use electrospray tandem mass spectrometry (ES-MS/MS) to investigate prodigiosin, undecylprodiginine, and streptorubin B (butyl-metacycloheptylprodiginine) and to explore their fragmentation pathways to explain the unusual methyl radical loss and consecutive fragment ions that dominate low-energy collision-induced dissociation (CID) mass spectra. The competition between the formation of even-electron ions and radical ions is examined in detail. Theoretical calculations are used to optimize the structures and calculate the energies of both reactants and products using the Gaussian 03 program. Results indicate that protonation occurs on the nitrogen atom that initially held no hydrogen, thus allowing formation of a pseudo-seven-membered ring that constitutes the most stable ground state $[\mathrm{M}+\mathrm{H}]^{+}$structure. From this precursor, experimental data show that methyl radical loss has the lowest apparent threshold but, alternatively, even-electron fragment ions can be formed by loss of a methanol molecule. Computational modeling indicates that methyl radical loss is the more endothermic process in this competition, but the lower apparent threshold associated with methyl radical loss points to a lower kinetic barrier. Additionally, this characteristic and unusual loss of methyl radical (in combination with weaker methanol loss) from each prodiginine is useful for performing constant neutral loss scans to quickly and efficiently identify all prodiginines in a complex biological mixture without any clean-up or purification. The feasibility of this approach has been proven through the identification of a new, low-abundance prodigiosin analog arising from Hahella chejuensis. (J Am Soc Mass Spectrom 2008, 19, 1856-1866) (c 2008 American Society for Mass Spectrometry

$\mathrm{P}$ odiginine analogs belong to a family of naturally occurring red-pigment antibiotics that are secondary metabolites biosynthesized by many strains of Gram-positive and Gram-negative bacteria [1]. These compounds are characterized by a common pyrrolydipyrrolylmethene backbone and a methoxy function on the B-ring (Scheme 1). The chemical composition of prodigiosin, a representative member of the prodiginine family, was established half a century ago by chemical synthesis $[2,3]$. Other close analogs, bearing the same skeleton but different alkyl substituents, have been reported, such as undecylprodiginine [4],

Address reprint requests to Dr. Richard B. Cole, University of New Orleans, Department of Chemistry, 2000 Lakeshore Dr., New Orleans, LA 70148, USA. E-mail: rcole@uno.edu streptorubin B [5], methylcyclodecylprodiginine [6], and cycloprodigiosin [7].

By virtue of their brilliant red color, these compounds were used as ink for a short time period before the appearance of synthetic dyes [8]. The prodiginines are also antibiotics that have a broad range of activities against bacteria, pathogenic fungi, and protozoa, but they have not been widely used in clinical trials because of their toxicity $[9,10]$. Some studies have shown that their common skeletal features, e.g., the presence of the B-ring C6-methoxy group (Scheme 1), are related to the cytotoxicity of prodigiosin; the A-ring also plays an important role in nuclease activity and cytotoxic effects [11]. The toxic effects were speculated to arise from intercalation with DNA, leading to oxidative DNA cleavages in the presence of $\mathrm{O}_{2}$ and $\mathrm{Cu}$ (II) $[12,13]$. Although the exact cytotoxic mechanism was not com- 


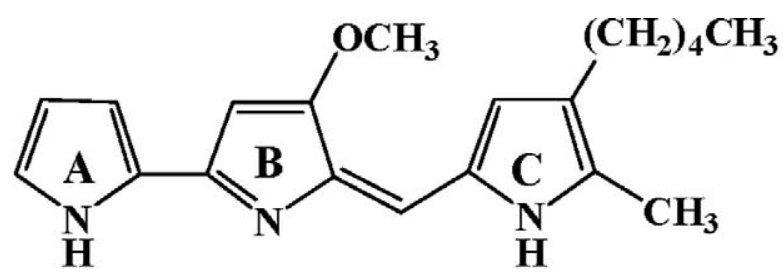

Prodigiosin

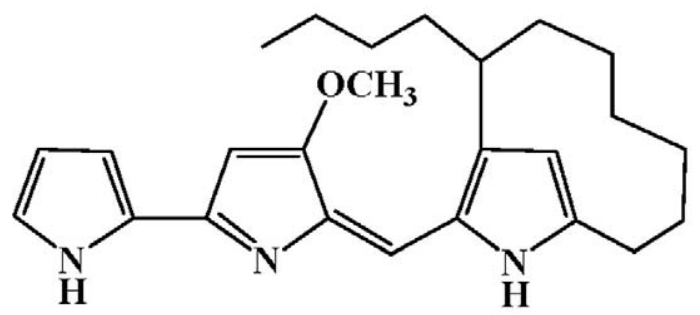

Streptorubin B

(Butyl-meta-cycloheptylprodiginine)

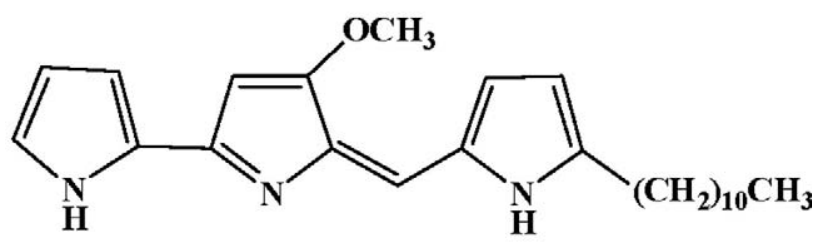

Undecylprodiginine

Scheme 1. Traditional structural representations of three investigated prodiginines: prodigiosin, streptorubin B, and undecylprodiginine.

pletely clarified, some recent findings have shown that prodiginine-type compounds are potent immunosuppressants at lower, nontoxic levels, and slightly higher dosages can efficiently induce apoptosis of cancer cells with little toxic effects on the normal cell lines in vivo and in vitro [14, 15].

Recent findings concerning their anticancer $[11,16]$ and immunosuppressive properties $[14,17,18]$ have led to the expansion of prodiginine studies. Intrigued by their pharmaceutical prospects, the total synthesis of prodiginine-type compounds has been reported [19]. Moreover, a synthetic analog named GX15-070 has been used in phase I/II clinical trials for cancer treatment [20]. However, other analogs anticipated to have even more antitumor activity, such as streptorubin B (butylmeta-cycloheptylprodiginine, Scheme 1), were difficult to synthesize in the lab. Thus, a biosynthetic pathway might be a good alternative to achieving rapid and cost-effective production of prodiginine-type drugs. To improve the understanding of the biosynthesis of prodiginine antibiotics, studies have been completed to elucidate pathways in Streptomyces coelicolor, leading to key intermediates including 4-methoxy-2,2'-bipyrrole5-carboxaldehyde (MBC) [21] and 2-undecylpyrrole (UP) [22].
Prodiginines show a characteristic brilliant red color and they readily absorb visible-region light that has been exploited for liquid chromatographic detection. Structural characterization has almost exclusively relied on nuclear magnetic resonance (NMR) spectroscopy that requires relatively large sample quantities, as well as labor-intensive purification steps. New developments in mass spectrometry over the last two decades have earned it the reputation for offering high sensitivity, a wealth of structural information with the use of complementary fragmentation techniques, and the ability to deal with complex mixtures. Up until now, however, mass spectrometry has mainly provided molecular weight confirmation in prodiginine identifications.

Understanding the major fragmentation pathways for prodiginines can help in the structural elucidation of synthetic prodiginine analogs and novel prodiginines generated by biosynthetic processes. Generally, all the prodiginine analogs are readily detected in the positiveion mode, because of available basic nitrogen atoms, which enables one to gain important structural information without further purification, especially if there are low-abundance prodiginine analogs in a complex mixture. Our study investigates all the major fragmentation mechanisms using multistage tandem mass spectrometry as well as theoretical calculations.

\section{Experimental}

\section{Chemicals and Sample Preparation}

Undecylprodiginine and streptorubin B were purified from Streptomyces coelicolor strains. Undecylprodiginine was isolated from mycelia of S. coelicolor W31, which is unable to make streptorubin B [23]. Streptorubin B was isolated from S. coelicolor M511, which produces a mixture of undecylprodiginine and streptorubin B. Mycelia were extracted with acetonitrile/methanol (50:50), acidified with $2 \mathrm{~N} \mathrm{HCl}(1 \%)$, and vortexed for $30 \mathrm{~s}$. One volume of chloroform and one volume of $1 \mathrm{~N} \mathrm{HCl}$ were then added to each volume of prepared extracts; and the chloroform fractions, which contained prodiginine hydrochlorides, were recovered. The fraction originating from S. coelicolor W31 was washed with $1 \mathrm{~N} \mathrm{NaOH}$ to afford undecylprodiginine as a free base that was purified by flash chromatography on basic alumina using $100 \%$ ethyl acetate as eluent. Undecylprodiginine appeared as a yellow pigment in ethyl acetate. The chloroform fraction originating from S. coelicolor M511 was evaporated to dryness and streptorubin B hydrochloride was purified by semipreparative HPLC using an Agilent 1100 instrument equipped with a quaternary pump and variable wavelength detector set at $533 \mathrm{~nm}$. Extracts that were resuspended in acetonitrile/methanol (50:50) were injected onto an Agilent C18 (Agilent Technologies, Santa Clara, CA, USA) column $(100 \times 21$ $\mathrm{mm}, 5 \mu \mathrm{m}$ ) using a gradient elution profile: water (adjusted to $\mathrm{pH} 3$ with $\mathrm{HCl}$ )/acetonitrile 30:70 to 20:80 
in $20 \mathrm{~min}$ at a flow rate of $5 \mathrm{~mL} / \mathrm{min}$; pure streptorubin B eluted after $8.2 \mathrm{~min}$.

In separate experiments, prodigiosin and its analog were extracted from Hahella chejuensis culture with a solvent of methanol containing $0.1 \mathrm{~N} \mathrm{HCl}$ on a shaker for $2 \mathrm{~h}$ at $4{ }^{\circ} \mathrm{C}$. The resultant mixture was centrifuged at $4000 \times g$ for $30 \mathrm{~min}$, and then the supernatant was collected.

\section{Mass Spectrometry}

ES-CID-MS $^{n}$ experiments were performed on a linear trapping quadrupole (LTQ) instrument (ThermoFinnigan, San Jose, CA, USA) equipped with a positive-ion nanospray ionization source and New Objective (Woburn, MA, USA) nanospray silica tips. Samples were diluted in $50 \%$ aqueous methanol containing $0.1 \%$ formic acid, and infusion loaded into the LTQ at a flow rate of 1 $\mu \mathrm{L} / \mathrm{min}$. The instrument was operated at a spray voltage of $2 \mathrm{kV}$ and a capillary temperature of $180^{\circ} \mathrm{C}$. Tandem mass spectra were acquired at a setting of $30 \%$ "normalized collision energy" of the precursor ions at $m / z=324.2,392.3$, and 394.3 corresponding to the three protonated prodiginines with an isolation width of 2.5 Da. Each MS and $\mathrm{MS}^{n}$ spectrum was acquired and averaged within $1 \mathrm{~min}$.

All neutral loss (NL) scans, and also all mass spectra appearing in Supplementary material, which can be found in the electronic version of this article, were performed on an Applied Biosystems/MDS SCIEX (Foster City, CA, USA) 3200 QTRAP hybrid quadrupole/ linear ion trap mass spectrometer with direct infusion of each prodiginine sample in aqueous methanol $(50 / 50)$ spiked with $0.1 \%$ formic acid at a flow rate of $4 \mu \mathrm{L} / \mathrm{min}$. Positive-ion mode ES-MS was used for the analysis, with the Turbo $\mathrm{V}^{\mathrm{TM}}$ source settings for prodiginines optimized as follows: ionspray voltage $5.5 \mathrm{kV}$, declustering potential $60 \mathrm{~V}$, source temperature $120^{\circ} \mathrm{C}$, GS1 40, and curtain gas 10 . Ultrahigh grade (99.999\%) nitrogen was utilized as nebulizing gas and collision gas. To maximize resolution of ionizable species in the complex sample, MS spectra were acquired using Q3 in the linear ion trapping (LIT) mode.

For the activation energy comparison using the 3200 QTRAP mass spectrometer, single-collision conditions ensured well-controlled energy deposition on the target ions during the ion activation event with precursor beam transmission $\geq 90 \%$. The ion abundances of the fragments from competing pathways were monitored as the collision energy was ramped from 5 to $80 \mathrm{eV}$. Each data point shown in the breakdown curve represents an average of five consecutive runs, and error bars (showing \pm standard deviation) were calculated from the five experiments.

\section{Computational Methods}

All computational calculations were performed using the Gaussian 03 (revision B.03) program [24]. The density functional B3PW91/6-31G* procedure (which combines the Becke three-parameter hybrid [25] and the Perdew-Wang correlation functionals [26]) was used to optimize geometries and these obtained structures were then used to find energy minima at $0 \mathrm{~K}$ [B3PW91/6$311+\mathrm{G}(2 \mathrm{~d}, 2 \mathrm{p})]$ with zero-point correction. When calculating transition states, opt $=$ QST2 and opt $=$ QST3 features were used with the relevant optimized reactants and products.

\section{Results and Discussion}

In positive-ion electrospray mass spectra, the ions observed are generally "molecular ions" representing intact forms of the analyte molecules; those created by proton attachment are denoted as $[\mathrm{M}+\mathrm{H}]^{+}$. These ions are even-electron $\left(\mathrm{EE}^{+}\right)$species that are generally much more stable than odd-electron ions $\left(\mathrm{OE}^{+\cdot}\right)$ formed, for example, by electron ionization. Even-electron ions such as protonated molecules formed by ES rarely decompose under low-energy collisions by homolytic cleavage involving loss of a radical to form $\mathrm{OE}^{+\cdot}+$ n (radical neutral) because the barrier to this type of process is almost always higher than that of competing processes. Rather, heterolytic bond cleavages, often involving rearrangement(s) [especially proton transfer(s)] reign. Thus, radical losses from $\mathrm{EE}^{+}$species are considered to be forbidden, which is a statement of the "even-electron rule" [27]. Certain exceptions to this "rule" have been reported where odd-electron ions were formed through the fragmentation of even-electron ions formed during electron ionization or chemical ionization or electrospray ionization [28-32]. These exceptions to the "even-electron rule" were proposed to involve radical eliminations leading to odd-electron fragment ions of exceptional stability [33].

The goal of the current study was to obtain a comprehensive view of the common fragmentation pathways of prodiginines, while mainly focusing on the odd-electron fragments observed in ES-tandem mass spectra. Even though three investigated prodiginine analogs share a common skeleton (Scheme 1), differences in the behavior of the protonated molecules following ion activation were seen as a result of the varying alkyl chain substituents on the C-ring.

\section{Interpretation of the Detailed Fragmentation Pathways of Prodigiosin}

Although the collision-induced dissociation (CID) mass spectra of some prodiginines have been reported [34], other than our preliminary presentation [35], to our knowledge there has been no detailed description of mass spectra, nor interpretation of their fragmentation mechanisms in the literature. In our CID mass spectra of three prodiginine analogs, similarities in the fragmen- 
tation patterns were readily discernable. In each case, a stable $\mathrm{OE}^{+\cdot}$ fragment ion is initially formed through the loss of a 15 Da neutral, which we deduce can only be a methyl radical. Methyl radical loss was also observed during the fragmentation of ES-generated protonated flavanones [31]. Because this $15 \mathrm{Da}$ loss is common to MS/MS spectra of all three prodiginines, we conclude that it must correspond to loss of the methyl moiety of the methoxy group on the B-ring $(\mathrm{m} / \mathrm{z} 324 \rightarrow 309, \mathrm{~m} / \mathrm{z}$ $392 \rightarrow 377, m / z \quad 394 \rightarrow 379$ in Figures 1, 2, and 3, respectively). Even though prodigiosin has another methyl substituent in the C-ring, neither of the other two prodiginines has a second methyl group; thus, C-ring methyl loss is eliminated as the source of $15 \mathrm{Da}$ loss. The fully conjugated skeletons provide much resonance stabilization for both the charge and the unpaired electron; when the latter is associated with the C-ring it can initiate cleavages on the substituent alkyl chains. To aid in the interpretation of the fragmentation mechanisms, CID-MS ${ }^{3}$ experiments were performed on the $\mathrm{OE}^{+\cdot}$ ions of particular interest (i.e., $m / z$ 309, 377, and 379 in Figures 1, 2, and 3, respectively). From these latter ions, obtained $\mathrm{MS}^{3}$ spectra could readily rationalize consecutive cleavages that appeared in the $\mathrm{MS}^{2}$ spectra. For example, it can be seen that alkyl chain cleavages yield the predominant fragment ions at $m / z$ 238 and 252 observed in the CID spectra of all three prodiginine analogs.

As a competing pathway, $\mathrm{EE}^{+}$fragments are derived from the loss of methanol $(\mathrm{m} / \mathrm{z} 324 \rightarrow 292, \mathrm{~m} / \mathrm{z} 392 \rightarrow$ $360, m / z 394 \rightarrow 362$ in Figures 1, 2, and 3, respectively). The ion abundance of this $\mathrm{EE}^{+}$product is substantially lower than that of the $\mathrm{OE}^{+\cdot}$ fragments $(\mathrm{m} / \mathrm{z} 309,377$, and 379 , respectively) observed in the tandem mass spectra, but clearly the methoxy group is involved, carrying a proton in tow in each case of methanol loss. Other $\mathrm{OE}^{+}$. fragments were also observed in the CID spectra of prodigiosin and its two analogs. Additional information to aid in elucidating the structures was gained using a third stage of tandem mass spectrometry. The $\mathrm{OE}^{+\cdot}$ products $[\mathrm{m} / \mathrm{z} 324 \rightarrow 149$ (Figure 1 ), $m / z 392 \rightarrow 295$ (Figure 3)] were further fragmented using on-resonance

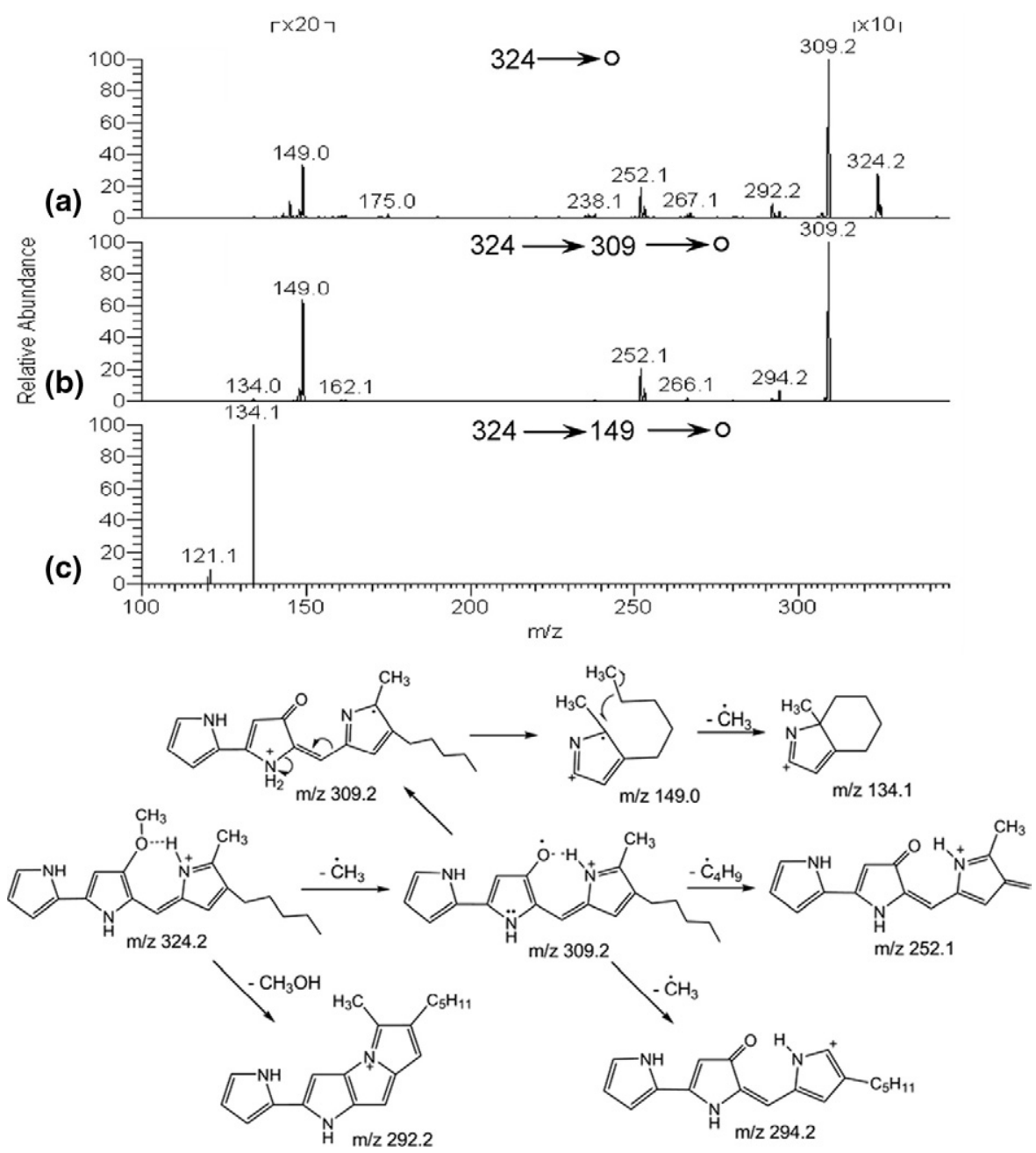

Figure 1. ES-CID-MS ${ }^{n}$ spectra for prodigiosin and the proposed major fragmentation pathways: (a) MS/MS spectrum of $[\mathrm{M}+\mathrm{H}]^{+}$precursor at $\mathrm{m} / \mathrm{z} 324 ;(\mathbf{b}) \mathrm{MS}^{3}$ spectrum of $\mathrm{m} / \mathrm{z} 309(324 \rightarrow 309)$; (c) MS $^{3}$ spectrum of $m / z 149(324 \rightarrow 149)$. 

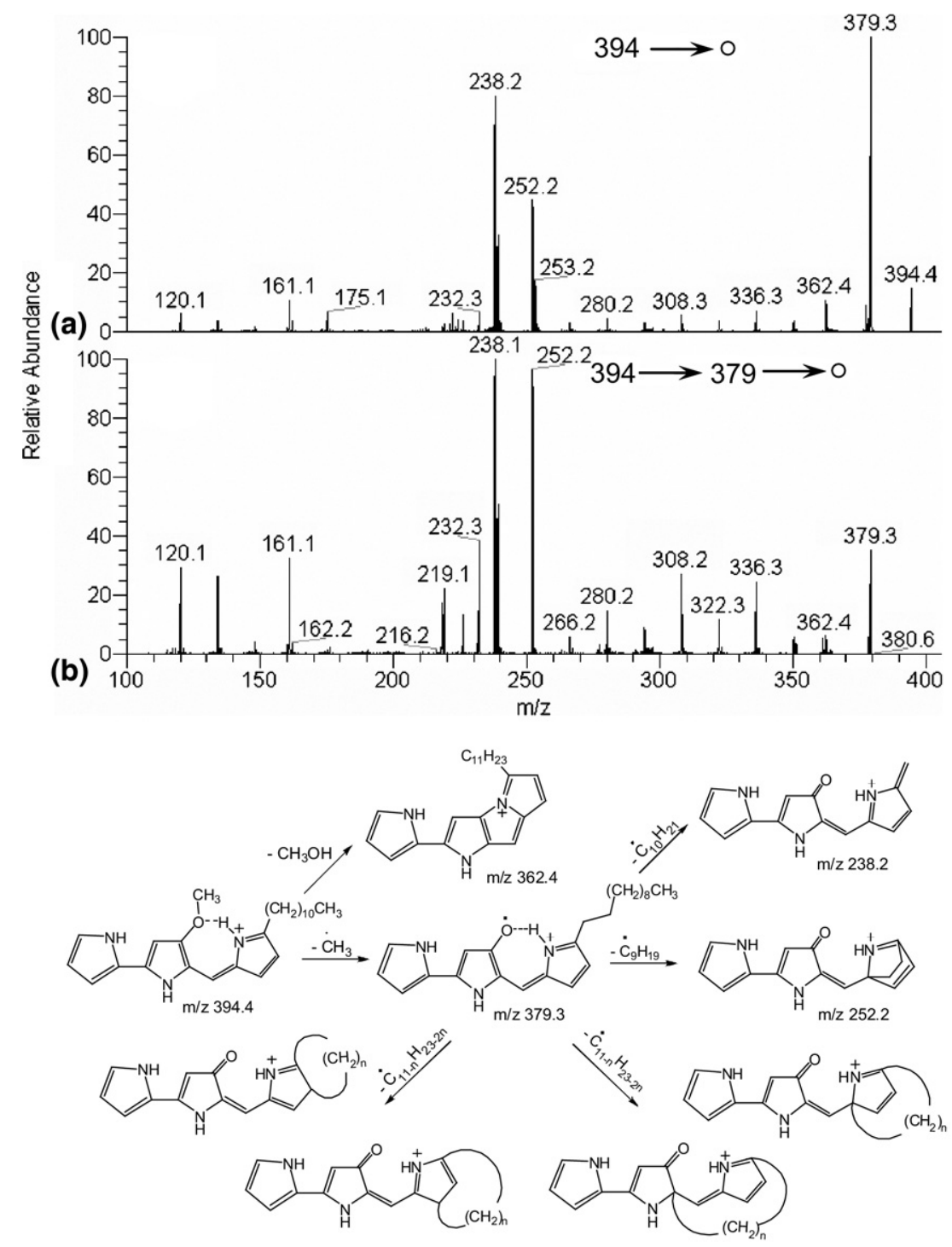

Figure 2. ES-CID-MS ${ }^{n}$ spectra for undecylprodiginine and the proposed principal fragmentation pathways: (a) MS/MS spectrum of $\left[\mathrm{M}+\mathrm{H}^{+}\right.$precursor at $\mathrm{m} / \mathrm{z} 394 ;(\mathbf{b}) \mathrm{MS}^{3}$ spectrum of $\mathrm{m} / \mathrm{z} 379$ (394 $\rightarrow 379$ ).

CID in the linear ion trap, giving major $\mathrm{EE}^{+}$fragments $\left(m / z 134\right.$ and 252, respectively) after $\mathrm{C}_{n} \mathrm{H}_{2 n+1}$ radical ( $n=1$ and 3 , respectively) elimination. The detailed mechanisms proposed for the fragmentations of the three investigated compounds are illustrated in Figures 1-3.

Additional evidence supporting these proposed mechanisms was obtained by repeating tandem mass spectrometry measurements, except that this time, a stable isotope labeled $\left[{ }^{13} \mathrm{C}\right.$-methoxy-undecylprodiginine + $\mathrm{H}]^{+}$m/z 395 precursor was used (see Supplemental material). All product ions appeared at exactly the same $\mathrm{m} / \mathrm{z}$ values as those derived from the unlabeled [undecylprodiginine $+\mathrm{H}^{+} \mathrm{m} / \mathrm{z} 394$ precursor. This result unambiguously implicates the methoxy function in both methyl radical and methanol losses and establishes that all other product ions arise from consecutive decompositions following one of these initial losses.

\section{Computational Studies of the Fragmentation Pathways of Prodiginines}

To investigate the underlying basis for the fragmentation pattern of protonated prodiginines, theoretical calculations were used to gain additional information concerning the competition between methyl radical and methanol losses. Density functional theory (DFT) calculations were performed to obtain the structures of the precursor ion and its decomposition products, transition states, and their associated energies. Because of the large numbers of atoms in the molecules of interest, it is time consuming to computationally minimize the energies of the structures using DFT, especially when a long alkyl chain with many approximately isoenergetic conformers is present. To reduce this difficulty, in all calculations, we used an ethyl analog of the undecyl- 

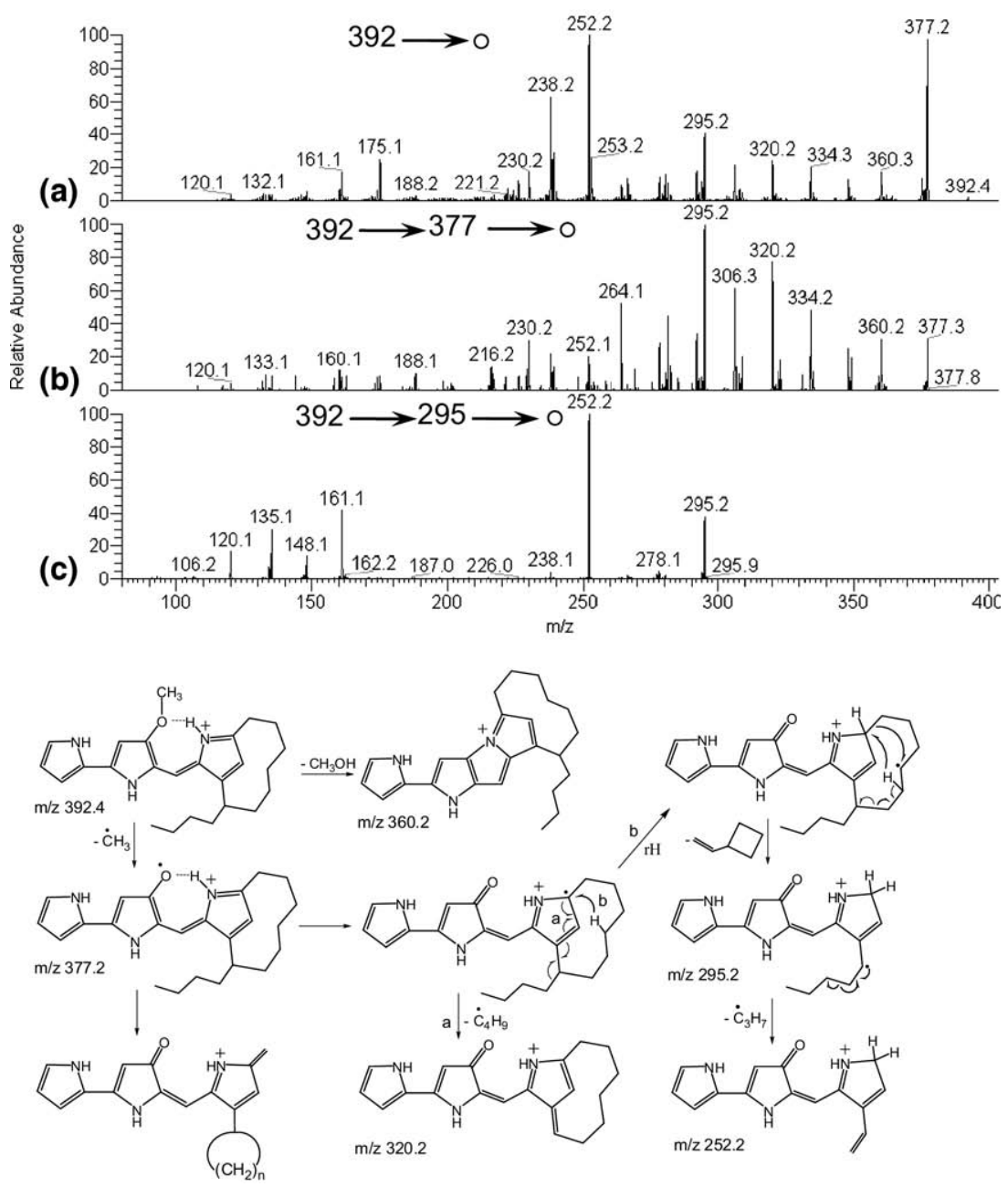

Figure 3. ES-CID-MS ${ }^{n}$ spectra for streptorubin B and the proposed major fragmentation pathways: (a) MS/MS spectrum of $[\mathrm{M}+\mathrm{H}]^{+}$precursor at $\mathrm{m} / \mathrm{z}$ 392; (b) $\mathrm{MS}^{3}$ spectrum of $\mathrm{m} / \mathrm{z} 377(392 \rightarrow 377)$; (c) $\mathrm{MS}^{3}$ spectrum of $m / z 295(392 \rightarrow 295)$.

prodiginine system, i.e., $\mathrm{C}_{2} \mathrm{H}_{5}$ substitution on the $\mathrm{C}$-ring instead of $\mathrm{C}_{11} \mathrm{H}_{23}$. Traditionally, the structures of prodiginines have been drawn with the three nitrogen atoms of the tripyrrole skeleton oriented on the same side of the molecule (Scheme 1). However, theoretical calculations find that the A- and C-rings in Figure 1 are rotated about $180^{\circ}$ such that the molecules are still nearly planar, but nitrogen lone pairs are located as far apart as possible to minimize the electronic repulsion and, thus, the system achieves the highest stability. Figure 4a I shows the energy optimized geometry obtained at the B3PW91/6-31G* level of theory, Figure 4a II shows the chemical structure, whereas Figure 4a III shows the electron density map. Electron density mapping provides insight into the electron distribution over the molecule and also allows prediction of the preferred site of proton attachment on the molecule. As can be seen in Figure 4a (III), the lone pair of electrons of the nitrogen atom in the B-ring exhibits the highest electron density and, thus, is clearly the preferred site of proton attachment. In view of the fully conjugated structure, the positive charge carried by the ionizing proton becomes highly delocalized over the entire ring system. In considering $[\mathrm{M}+\mathrm{H}]^{+}$ions observed in mass spectra, and placing the ionizing proton on the nitrogen that previously holds no proton, the energies of three stable conformers were calculated at the B3PW91/6-311+ $\mathrm{G}(2 \mathrm{~d}, 2 \mathrm{p})$ level of theory; the relative stabilization energies are listed below each corresponding structure (Figure $4 \mathrm{~b}$ ). Very importantly, a hydrogen bond may form between the C-ring proton attached to the nitrogen atom and the oxygen from the methoxy function to produce a pseudoseven-membered ring (Scheme 2), which represents the most stable ground state conformation.

With the proton held (chelated) between the C-ring nitrogen and the methoxy oxygen $(\mathrm{O} \cdots \mathrm{H}$ distance = $1.397 \AA$ ), two facile decomposition pathways are possible. The first pathway is the expected loss of a methanol molecule that, afterward, according to energy minimization studies, undergoes ring closure to form a new fused five-membered ring structure (Scheme 2). The second pathway is homolytic cleavage between the 
(a)

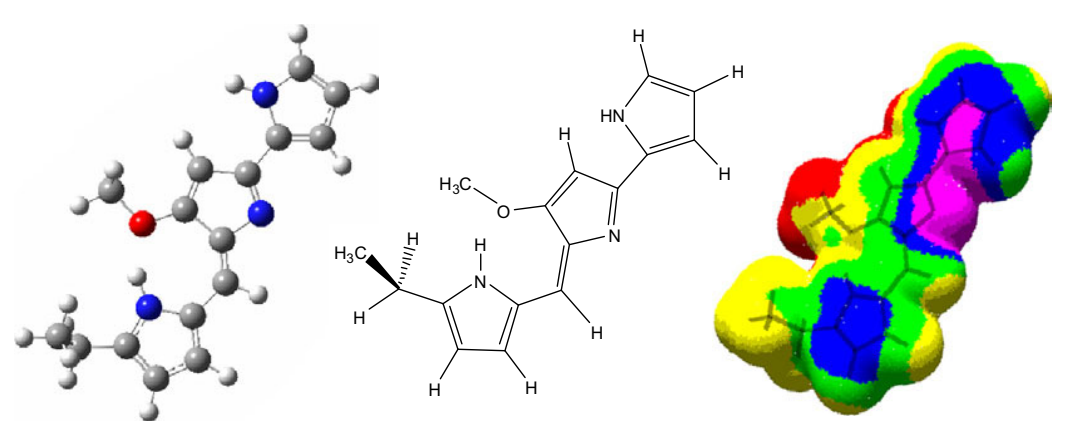

(b)

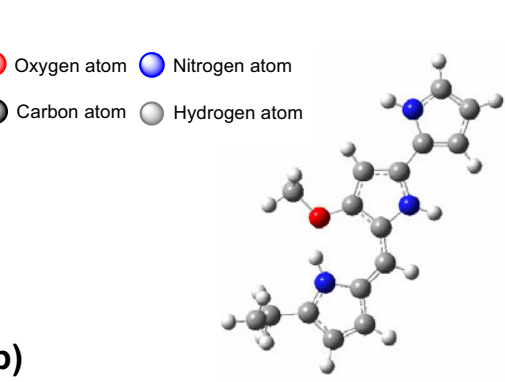

B3PW91/6-311+G(2d,2p) $\quad 0.0 \mathrm{~kJ} / \mathrm{mol}$

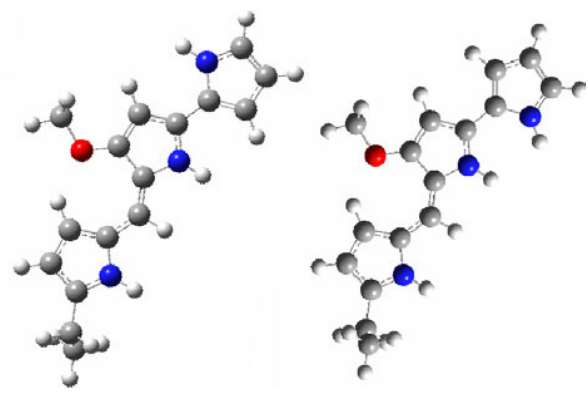

$6.1 \mathrm{~kJ} / \mathrm{mol}$

$12.4 \mathrm{~kJ} / \mathrm{mol}$

Figure 4. (a) Structures of neutral prodiginine model system showing the preferred site for proton attachment during the electrospray ionization process. (I) the energetically optimized geometry at the B3PW91 /6-31G* level; (II) chemical structure; (III) electron density map (order of decreasing electron density purple $>$ blue $>$ green $>$ yellow $>$ red) indicating highest electron density at the central nitrogen bearing no proton. (b) The optimized prodiginine conformations and their relative energies calculated by density function theory (DFT).

oxygen and carbon atoms of the methoxy group to produce loss of $\mathrm{CH}_{3}$, which was experimentally observed to be surprisingly facile and favored.

Geometry optimization and vibrational frequency analysis for the reactant and the two products were carried out at the B3PW91/6-31G* level. These structures were then used to perform single-point energy calculations at the B3PW91/6-311+G(2d,2p) level with zeropoint energy correction. The calculated relative energies of the two endothermic products are 121.6 (EE ${ }^{+}$product) and $226.8\left(\mathrm{OE}^{+\cdot}\right.$ product) $\mathrm{kJ} / \mathrm{mol}$ (Scheme 2), rendering methyl loss more endothermic by $105.2 \mathrm{~kJ} / \mathrm{mol}$. Thus, the preference for methyl radical loss over methanol loss cannot be explained by the energy difference between the reactant and the respective products.

\section{Comparison of Apparent Thresholds and Activation Energies for the Two Competing Mechanisms}

To obtain more insight into the competing processes of methyl radical loss versus methanol loss from proton-

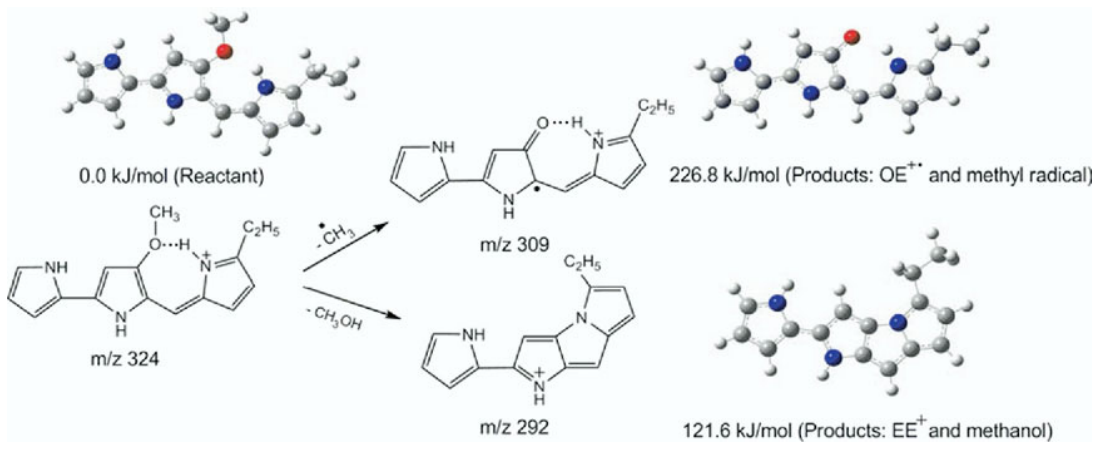

Scheme 2. Two competing fragmentation pathways of the protonated prodiginine model system leading either to $\mathrm{OE}^{+\cdot}$ after homolytic dissociation to lose a methyl radical, or to $\mathrm{EE}^{+}$after heterolytic bond cleavage to lose a methanol molecule. 
ated prodiginines, a profiling of the fragment ion populations from each pathway as a function of increasing energy under single-collision conditions was undertaken in the low-energy regime. Figure 5a presents the breakdown curve of the protonated prodigiosin precursor $(m / z 324)$, plus the appearance of the fragments associated with methyl radical loss $(\mathrm{m} / \mathrm{z} 309$ and 252) and methanol loss ( $m / z$ 292). Most notably, methyl radical loss has the lowest apparent threshold $\left(\mathrm{E}_{\mathrm{lab}} \approx 5 \mathrm{eV}\right)$ and its consecutive fragmentation pathway (giving $m / z$ 252, Figure 1) appears instantaneously thereafter. Methanol loss exhibits a higher apparent threshold $\left(\mathrm{E}_{\mathrm{lab}} \approx 12.5 \mathrm{eV}\right)$ compared to the former loss. Surprisingly, Figure 5a shows that, regardless of collision energy, the abundances of $m / z 309$ and 252 are always more than 10 times higher than that of $m / z$ 292. MS/MS spectra that were acquired at collision energies of 5 and $12.5 \mathrm{eV}$ $\left(\mathrm{E}_{\text {lab }}\right)$ are shown in Figure $5 b$.
Methyl radical loss thus has a lower apparent threshold, but it is also a more endothermic pathway than methanol loss. This implies that $\mathrm{OE}^{+\cdot}$ production is favored because methyl radical loss presents the lowest available kinetic barrier, which entails a low reverse activation barrier. Computational studies were used in an attempt to calculate activation energies and a transitionstate structure for loss of methanol from protonated prodiginine with only one imaginary frequency was successfully obtained. The activation energy for loss of methanol from protonated prodiginine was calculated to pass through a transition state that was found to be 373.3 $\mathrm{kJ} / \mathrm{mol}$ higher in energy than the ground state reactant. Unfortunately, our attempt to obtain a transition state for methyl radical loss from protonated prodiginine was unsuccessful; nonetheless, the experimental data in Figure 5 prove that the energy barrier for this latter process is significantly lower than that of methanol loss.

(a)
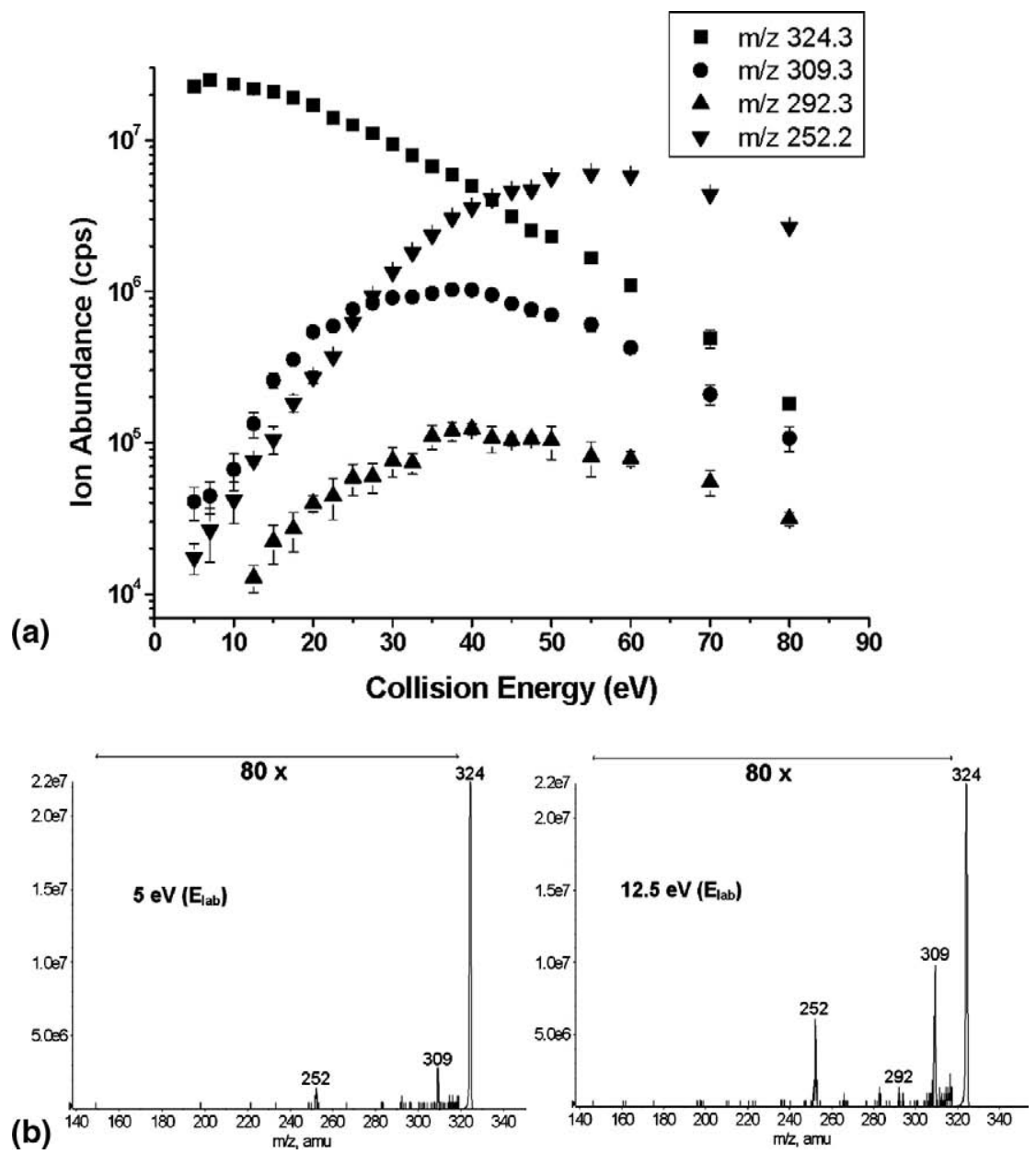

Figure 5. (a) Breakdown curves showing ion abundance versus collision energy (eV) for MS/MS of prodigiosin. The plots show the evolution of precursor and fragment ions representing two competing pathways $\left(\mathrm{m} / \mathrm{z} 324=\right.$ precursor; $\mathrm{m} / \mathrm{z} 309$ and 252 represent the formation of $\mathrm{OE}^{+\cdot} \cdot$ by methyl radical loss and methyl radical loss plus consecutive butyl radical loss, respectively; $m / z 292$ represents the formation of $\mathrm{EE}^{+}$after the loss of methanol). (b) The MS/MS spectra of the $m / z 324$ prodigiosin precursor with collision energies of 5,10 , and $12.5 \mathrm{eV}$ performed under single collision conditions. 


\section{New Strategy for Prodiginine Identification}

Because of the distinct and unusual nature of the fragments resulting from the competing losses of either methyl radical or methanol, we decided to make an attempt to analytically exploit this uniqueness and use the combination of the two losses as a characteristic marker for prodiginine-type compounds. The instrument design of the QTRAP mass spectrometer enables neutral loss (or gain) scans of all ions generated in the ion source, thus enabling a quick screening of all the analytes present in a crude mixture that undergo a specific type of decomposition. Neutral loss scans of 15
$\mathrm{Da}$ (representing a methyl radical) and $32 \mathrm{Da}$ (representing a methanol molecule) were performed using a crude sample extract from $H$. chejuensis in which prodigiosin was previously identified using preparative LC separation and NMR spectroscopy [34]. The $15 \mathrm{Da}$ neutral loss scan detected not only prodigiosin, but also a new, low-abundance prodigiosin analog from the crude mixture (no purification step). A subsequent 32 Da neutral loss scan confirmed the presence of this prodigiosin analog. These results are summarized schematically in Figure 6. The ES mass spectrum (Figure 6a) reveals protonated prodigiosin as the base peak at $\mathrm{m} / \mathrm{z}$
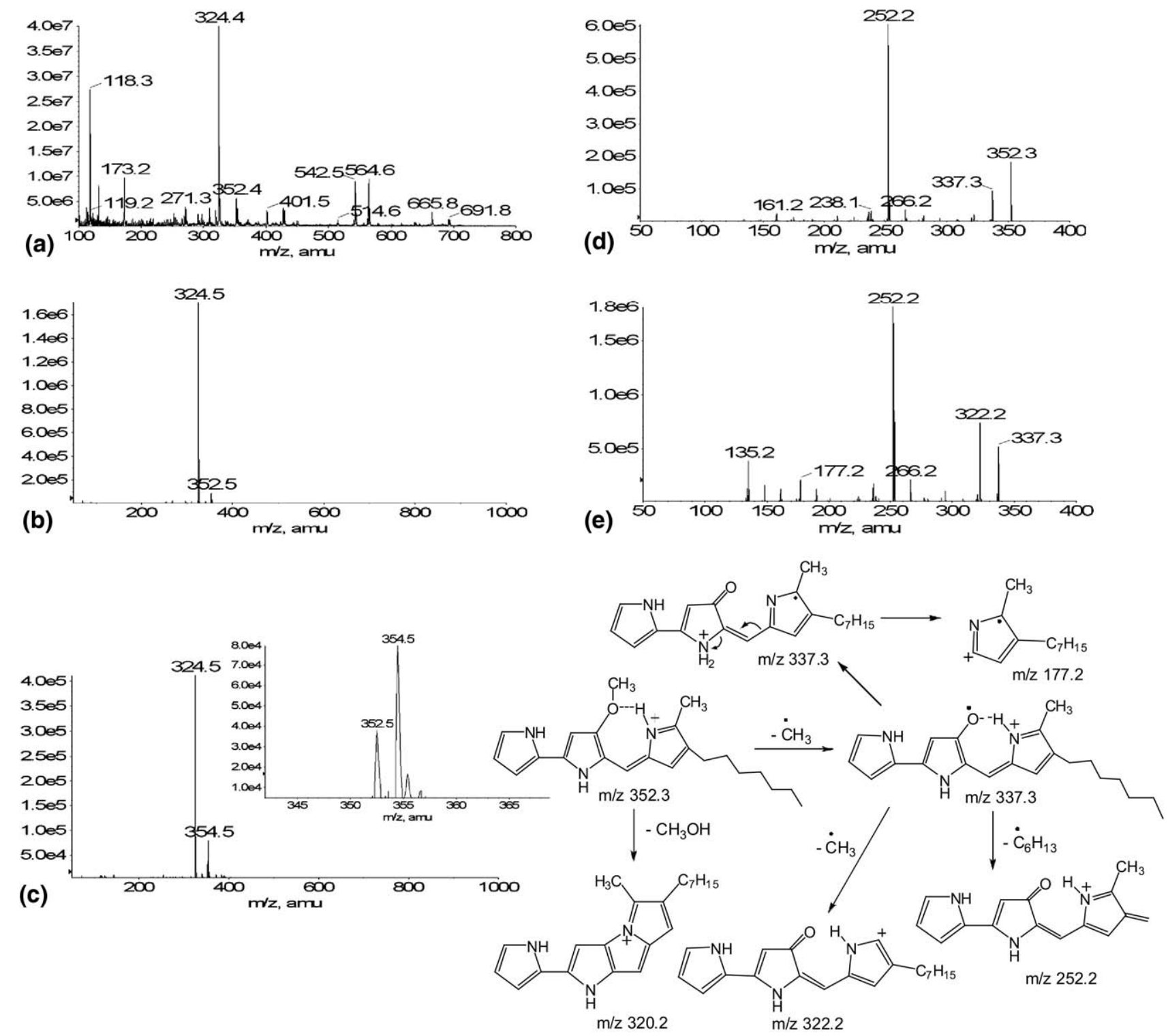

Figure 6. (a) Positive-ion ES-MS spectrum of the extract from $H$. chejuensis showing $[\mathrm{M}+\mathrm{H}]^{+}$of $m / z$ 324 from prodigiosin, and $\mathrm{m} / \mathrm{z} 352$ from the analog; (b) positive-ion neutral loss (15 Da) spectrum, showing both prodigiosin and its analog at $m / z 324$ and 352, respectively; (c) positive-ion neutral loss spectrum of 32 Da mass loss, showing both prodigiosin and its analog at $\mathrm{m} / z 324$ and 352 (expanded in inset); (d) positive-ion ES-MS/MS spectrum of protonated prodigiosin analog at $\mathrm{m} / \mathrm{z} 352$; and (e) $\mathrm{MS}^{3}$ spectrum of $\mathrm{m} / \mathrm{z} 337$; the proposed fragmentation pathways of protonated prodigiosin analog at $m / z 352$. 
324. Figure $6 b$ and $c$ show the neutral loss mass spectra corresponding to methyl radical and methanol losses, respectively, in which both prodigiosin $(\mathrm{m} / \mathrm{z} 324)$ and its analog $(m / z$ 352) appeared.

The characterization of the analog at $m / z 352$ is based on its tandem mass spectra (Figure $6 \mathrm{~d}$ and e). Like the other prodiginines, it shows fragments resulting from various alkyl chain cleavages. The pathways leading to the observed fragments are proposed at the bottom of Figure 6. In the fragmentation mechanism of prodigiosin (Figure 1), we proposed that the product ion at $\mathrm{m} / \mathrm{z}$ 252 was generated from cleavage of the alkyl chain on the C-ring. The proposed pathway to form the same fragment at $m / z 252$ (in Figure 6d and e) indicates that the analog shares the same backbone as prodigiosin (including the methyl group on the C-ring) but differs in the nature of the second alkyl chain. The presence of a (second) methyl loss (in Figure 6e) leading to $\mathrm{m} / \mathrm{z} 322$ further supports the notion that, similar to prodigiosin, a methyl group exists on the C-ring. Thus, the evidence suggests that the mass difference of $28 \mathrm{Da}$ between the two compounds reflects the extension of the longer alkyl chain on the C-ring of prodigiosin by two $-\mathrm{CH}_{2}-$ units. Additional evidence is provided by the fragment ion at $m / z 177$ derived from the precursor at $m / z 337$ (Figure 6e) that was generated from the same pathway as the fragment at $m / z 149$ in the tandem mass spectrum of prodigiosin (Figure 1). This mass difference of $28 \mathrm{Da}$ between these two fragments confirms the extension of the alkyl chain substitution on the C-ring. However, one cannot determine by mass spectrometry alone, whether or not the alkyl chain is branched. Thus, we identified the analog as containing a $\mathrm{C}_{7} \mathrm{H}_{15}$ saturated alkyl chain on the C-ring instead of $\mathrm{C}_{5} \mathrm{H}_{11}$ in prodigiosin [34]. Because prodigiosin and undecylprodiginine both contain $n$-alkyl chains, we assign the new analog as also being unbranched.

The studies described herein have thus proven that our approach of using a combination of constant neutral loss scans (15 and $32 \mathrm{Da}$ ) is selective for the detection of unknown and low-abundance prodiginine compounds; the strategy is built on the ease of loss of methyl radicals from their common backbone structure. Without laborintensive purification steps, the workload for identification and characterization of prodiginines can thus dramatically decrease using our approach. Because of the excellent sensitivity of mass spectrometry, the sample quantity requirements could also decrease from milligrams (for NMR) to less than micrograms (for mass spectrometry) of a crude mixture.

\section{Conclusion}

The fragmentation behavior in positive-mode electrospray mass spectrometry of three representative members of prodiginines that share the same pyrrolydipyrrolylmethene skeleton has been investigated. Two competing fragmentation pathways were the loss of either methyl radical or methanol and, in violation of the even-electron rule, the CID spectra were dominated by the former pathway to yield $\mathrm{OE}^{+}$products that may undergo consecutive fragmentations. Upon structural optimization of reactant $\mathrm{MH}^{+}$precursors using a Gaussian 03 computational program, results indicate that the nitrogen atom at the C-ring and the B-ring methoxy function could effectively chelate a proton to form a relatively stable $\mathrm{H}$-bonded pseudo-seven-membered ring. From this precursor, methyl radical loss is the most facile decomposition pathway, as evidenced by its dominance in low-energy tandem mass spectra. In a competitive pathway, the loss of a methanol molecule involving the same methoxy site on the precursor to produce an $\mathrm{EE}^{+}$fragment was heavily disfavored. The apparent threshold of the former pathway has been shown to be much lower than the latter by profiling the ion population of the major fragments of protonated prodigiosin against collision energy under singlecollision conditions. Thus, the fragmentation of prodiginines was kinetically dominated by methyl loss over methanol loss as a first step. Moreover, the characteristic and unusual loss of methyl radical from each prodiginine, in favorable competition with methanol loss, is useful for rapid identification and structural elucidation of novel prodiginines by performing constant neutral loss scans $\left(\cdot \mathrm{CH}_{3}, 15 \mathrm{Da}\right.$ and $\mathrm{CH}_{3} \mathrm{OH}, 32$ $\mathrm{Da})$ to identify all prodiginines present in a mixture.

Using analytical methodology built on this strategy, we report a new and low-abundance prodigiosin ana$\log$ from $H$. chejuensis. Because methyl radical loss rarely occurs in the fragmentation of most ES-generated compounds ( $\mathrm{EE}^{+}$species), the selectivity of the method will permit high-sensitivity identifications of prodiginines from crude extracts of different bacterial species.

\section{Acknowledgments}

Financial support for this work was provided by the National Science Foundation through CHE-0518288 and CHE-0630427, by the Louisiana Board of Regents through HEF (2001-06)-08, and by the European Union (Integrated Project Actinogen, Contract 005224).

\section{References}

1. Williams, R. P. Biosynthesis of Prodigiosin, a Secondary Metabolite of Serratia marcescens. Appl. Microbiol. 1973, 25, 7102-7109.

2. Wasserman, H. H.; McKeon, J.; Santer, U. V. Studies Related to the Biosynthesis of Prodigiosin in Serratia marcescens. Biochem. Biophys. Res. Commun. 1960, 3, 146-149.

3. Rapoport, H.; Holden, K. G. The Synthesis of Prodigiosin. J. Am. Chem. Soc. 1962, 82, 5510-5511.

4. Gerber, N. N. Prodigiosin-like Pigments. CRC Crit. Rev. Microbiol. 1975, 3, 469-485

5. Laatsch, H.; Kellner, M.; Weyland, H. Butyl-meta-cycloheptylprodiginine - a revision of the structure of the former ortho-isomer, J. Antibiot. 1991, 44, 187-191.

6. Gerber, N. N. Prodigiosin-like Pigments from Actinomadura (Nocardia) pelletieri. J. Antibiot. 1971, 24, 636-640.

7. Kawauchi, K.; Shibutani, K.; Yagisawa, H.; Kamata, H.; Nakatsuji, S. Anzai, H.; Yokoyama, Y.; Ikegami, Y.; Moriyama, Y.; Hirata, H. A Possible Immunosuppressant, Cycloprodigiosin Hydrochloride, Obtained from Pseudoalteromonas denitrificans. Biochem. Biophys. Res. Commun. 1997, 237, 543-547.

8. Bennett, J. W.; Bentley, R. Seeing Red: The Story of Prodigiosin. Adv. Appl. Microbiol. 2000, 47, 1-32. 
9. Furstner, A. Chemistry and Biology of Roseophilin and the Prodigiosin Alkaloids: A Survey of the Last 2500 Years. Angew. Chem. Int. Ed. Engl. 2003, 42, 3582-3603.

10. Cerdeno, A. M.; Bibb, M. J.; Challis, G. L. Analysis of the Prodiginine Biosynthesis Gene Cluster of Streptomyces coelicolor A3(2): New Mechanisms for Chain Initiation and Termination in Modular Multienzymes. Chem. Biol. 2001, 8, 817-829.

11. Llagostera, E.; Soto-Cerrato, V.; Montaner, B.; Perez-Tomas, R. Prodigiosin Induces Apoptosis by Acting on Mitochondria in Human Lung Cancer Cells. Ann. N. Y. Acad. Sci. 2003, 1010, 178-181.

12. Manderville, R. A. Synthesis, Proton-Affinity and Anti-Cancer Properties of the Prodigiosin-Group Natural Products. Curr. Med. Chem. Anticancer Agents 2001, 1, 195-218.

13. Montaner, B.; Castillo-Avila, W.; Martinell, M.; Ollinger, R.; Aymami, J.; Giralt, E.; Perez-Tomas, R. DNA Interaction and Dual Topoisomerase I and II Inhibition Properties of the Anti-Tumor Drug Prodigiosin. Toxicol. Sci. 2005, 85, 870-879.

14. Perez-Tomas, R.; Montaner, B.; Llagostera, E.; Soto-Cerrato, V. The Prodigiosins, Proapoptotic Drugs with Anticancer Properties. Biochem. Pharmacol. 2003, 66, 1447-1452.

15. Montaner, B.; Perez-Tomas, R. The Prodigiosins: A New Family of Anticancer Drugs. Curr. Cancer Drug Targets 2003, 3, 57-65.

16. Soto-Cerrato, V.; Llagostera, E.; Montaner, B.; Scheffer, G. L.; PerezTomas, R. Mitochondria-Mediated Apoptosis Operating Irrespective of Multidrug Resistance in Breast Cancer Cells by the Anticancer Agent Prodigiosin. Biochem. Pharmacol. 2004, 68, 1345-1352.

17. Stepkowski, S. M.; Nagy, Z. S.; Wang, M. E.; Behbod, F.; Erwin-Cohen, R.; Kahan, B. D.; Kirken, R. A. PNU156804 Inhibits Jak3 Tyrosine Kinase and Rat Heart Allograft Rejection. Transplant. Proc. 2001, 33, 3272-3273.

18. Mortellaro, A.; Songia, S.; Gnocchi, P.; Ferrari, M.; Fornasiero, C.; D’Alessio, R.; Isetta, A.; Colotta, F.; Golay, J. New Immunosuppressive Drug PNU156804 Blocks IL-2-dependent Proliferation and NF-kappa B and AP-1 Activation. J. Immunol. 1999, 162, 7102-7109.

19. Furstner, A.; Grabowski, J.; Lehmann, C. W. Total Synthesis and Structural Refinement of the Cyclic Tripyrrole Pigment Nonylprodigiosin. J. Org. Chem. 1999, 64, 8275-8280.

20. Dairi, K.; Tripathy, S.; Attardo, G.; Lavallee, J. F. Two Step Synthesis of the Bipyrrole Precursor of Prodigiosins. Tetrahedron Lett. 2006, 47, 2605-2606.

21. Stanley, A. E.; Walton, L. J.; Kourdi Zerikly, M.; Corre, C.; Challis, G. L. Elucidation of the Streptomyces coelicolor Pathway to 4-Methoxy-2,2' bipyrrole-5-carboxaldehyde, an Intermediate in Prodiginine Biosynthesis. Chem. Commun. (Camb.) 2006, 38, 3981-3983.

22. Mo, S. J.; Sydor, P. K.; Corre, C.; Alhamadsheh, M. M.; Stanley, A. E.; Haynes, S. W.; Song, L.; Reynolds, K. A.; Challis, G. L. Elucidation of the Streptomyces coelicolor Pathway to 2-Undecylpyrrole, a Key Intermediate in Undecylprodiginine and Streptorubin B Biosynthesis. Chem. Biol. 2008, 15, 137-148.

23. Odulate, O.; Barona-Gomez, F.; Corre, C.; Challis, G. L. Unpublished results.

24. Frisch, M. J.; Trucks, G. W.; Schlegel, H. B.; Scuseria, G. E.; Robb, M. A.; Cheeseman, J. R.; Montgomery, J. A. J.; Vreven, T.; Kudin, K. N.; Burant, J. C.; Millam, J. M.; Iyengar, S. S.; Tomasi, J.; Barone, V.; Mennucci, B.; Cossi, M.; Scalmani, G.; Rega, N.; Petersson, G. A.; Nakatsuji, H.; Hada,
M.; Ehara, M.; Toyota, K.; Fukuda, R.; Hasegawa, J.; Ishida, M.; Nakajima, T.; Honda, Y.; Kitao, O.; Nakai, H.; Klene, M.; Li, X.; Knox, J. E.; Hratchian, H. P.; Cross, J. B.; Bakken, V.; Adamo, C.; Jaramillo, J.; Gomperts, R.; Stratmann, R. E.; Yazyev, O.; Austin, A. J.; Cammi, R.; Pomelli, C.; Ochterski, J. W.; Ayala, P. Y.; Morokuma, K.; Voth, G. A.; Salvador, P.; Dannenberg, J. J.; Zakrzewski, V. G.; Dapprich, S.; Daniels, A. D.; Strain, M. C.; Farkas, O.; Malick, D. K.; Rabuck, A. D. Raghavachari, K.; Foresman, J. J. B.; Ortiz, V.; Cui, Q.; Baboul, A. G.; Clifford, S.; Cioslowski, J.; Stefanov, B. B.; Liu, G.; Liashenko, A. Piskorz, P.; Komaromi, I.; Martin, R. L.; Fox, D. J.; Keith, T.; Al-Laham, M. A.; Peng, C. Y.; Nanayakkara, A.; Challacombe, M.; Gill, P. M. W. Johnson, B.; Chen, M. W.; Wong, W.; Gonzalez, C. Gaussian 03, Revision B. 03; Gaussian Inc,: Pittsburgh, PA, 2003.

25. Becke, A. D. Density-Functional Thermochemistry. III. The Role of Exact Exchange. J. Chem. Phys. 1993, 98, 5648-5652.

26. Perdew, J. P.; Wang, Y. Pair-Distribution Function and Its CouplingConstant Average for the Spin-Polarized Electron Gas. Phys. Rev. B 1992, $46,12947$.

27. Rajkarnikar, A.; Kwon, H. J.; Suh, J. W. Role of Adenosine Kinase in the Control of Streptomyces Differentiations: Loss of Adenosine Kinase Suppresses Sporulation and Actinorhodin Biosynthesis While Inducing Hyperproduction of Undecylprodigiosin in Streptomyces lividans. Biochem. Biophys. Res. Commun. 2007, 363, 322-328.

28. Sigsby, M. L.; Day, R. J.; Cooks, R. G. Fragmentation of Even Electron Ions: Protonated Ketones and Ethers. Org. Mass. Spectrom. 1979, 14, 273

29. Fenselau, C.; Brown, P.; Patterson, D. G. Fragmentation of $[\mathrm{M}+\mathrm{H}]^{+}$to Form Ion Radicals Following Chemical Ionization. Spectroscopy (Amsterdam) 1983, 2, 348-351.

30. Bowie, J. H.; Stringer, M. B.; Duus, F.; Lawesson, S. O.; Larsson, F. C. V.; Madsen, J. O. Fragmentations of Organic Negative-Ions, Mercapto Acids and Esters: A Reinvestigation. Aust. J. Chem. 1984, 37, 1619-1624.

31. Nikolic, D. Li, Y. Chadwick, L. R. Grubjesic, S. Schwab, P. Metz, P. van Breemen, R. B. Metabolism of 8-Prenylnaringenin, a Potent Phytoestrogen from Hops (Humulus lupulus), by Human Liver Microsomes. Drug Metab. Dispos. 2004, 32, 272-279.

32. Levsen, K.; Schiebel, H. M.; Terlouw, J. K.; Jobst, K. J.; Elend, M.; Preiss, A.; Thiele, H.; Ingendoh, A. Even-Electron Ions: A Systematic Study of the Neutral Species Lost in the Dissociation of Quasi-Molecular Ions. J. Mass Spectrom. 2007, 42, 1024-1044.

33. Williams, J. P.; Nibbering, N. M.; Green, B. N.; Patel, V. J.; Scrivens, J. H. Collision-Induced Fragmentation Pathways Including Odd-Electron Ion Formation from Desorption Electrospray Ionisation Generated Protonated and Deprotonated Drugs Derived from Tandem Accurate Mass Spectrometry. J. Mass Spectrom. 2006, 41, 1277-1286.

34. Jeong, H.; Yim, J. H.; Lee, C.; Choi, S. H.; Park, Y. K.; Yoon, S. H.; Hur C. G.; Kang, H. Y.; Kim, D.; Lee, H. H.; Park, K. H.; Park, S. H.; Park, H. S.; Lee, H. K.; Oh, T. K. Kim, J. F. Genomic Blueprint of Hahella chejuensis, a Marine Microbe Producing an Algicidal Agent. Nucleic Acids Res. 2005, 33, 7066-7073.

35. Chen, K.; Lane, P.; Cai, Y.; Rees, B. B.; Challis, G. L.; Cole R. B. Unusual Odd-Electron Fragments from Even-Electron Protonated Prodiginine Precursors Using Positive Ion Electrospray Tandem Mass Spectrometry. Proceedings of the $55^{\text {th }}$ ASMS Conference on Mass Spectrometry and Allied Topics, Indianapolis IN, June 3-7, 2007. 